\title{
DNIC-mediated analgesia produced by a supramaximal electrical or a high-dose formalin conditioning stimulus: roles of opioid and $\alpha 2$-adrenergic receptors
}

Yeong-Ray Wen ${ }^{1,2,3}$, Chia-Chuan Wang ${ }^{4}$, Geng-Chang Yeh ${ }^{1}$, Sheng-Feng Hsu' ${ }^{5}$ Yung-Jen Huang ${ }^{3}$, Yen-Li Li ${ }^{3}$, Wei-Zen Sun ${ }^{*}$

\begin{abstract}
Background: Diffuse noxious inhibitory controls (DNIC) can be produced by different types of conditioning stimuli, but the analgesic properties and underlying mechanisms remain unclear. The aim of this study was to differentiate the induction of DNIC analgesia between noxious electrical and inflammatory conditioning stimuli.

Methods: First, rats subjected to either a supramaximal electrical stimulation or an injection of high-dose formalin in the hind limb were identified to have pain responses with behavioral evidence and spinal Fos-immunoreactive profiles. Second, suppression of tail-flick latencies by the two noxious stimuli was assessed to confirm the presence of DNIC. Third, an opioid receptor antagonist (naloxone) and an $\alpha 2$-adrenoreceptor antagonist (yohimbine) were injected, intraperitoneally and intrathecally respectively, before conditioning noxious stimuli to test the involvement of descending inhibitory pathways in DNIC-mediated analgesia.

Results: An intramuscular injection of $100 \mu$ of $5 \%$ formalin produced noxious behaviors with cumulative pain scores similar to those of $50 \mu \mathrm{l}$ of $2 \%$ formalin in the paw. Both electrical and chemical stimulation significantly increased Fos expression in the superficial dorsal horns, but possessed characteristic distribution patterns individually. Both conditioning stimuli prolonged the tail-flick latencies indicating a DNIC response. However, the electrical stimulation-induced DNIC was reversed by yohimbine, but not by naloxone; whereas noxious formalininduced analgesia was both naloxone- and yohimbine-reversible.
\end{abstract}

Conclusions: It is demonstrated that DNIC produced by different types of conditioning stimuli can be mediated by different descending inhibitory controls, indicating the organization within the central nervous circuit is complex and possibly exhibits particular clinical manifestations.

\section{Background}

Nociception is dynamically regulated by endogenous modulation systems, and final pain perception depends on a balance between nociceptive stimulation and the processing networks. Diffuse noxious inhibitory controls (DNIC), among the networks, occur when a painful stimulus (i.e., a conditioning stimulus) in one body region suppresses another noxious response (i.e., a test stimulus) in a remote body region $[1,2]$, and is an important

\footnotetext{
* Correspondence: wzsun@ntu.edu.tw
${ }^{6}$ Department of Anesthesiology, National Taiwan University Hospital, Taipei,

* Correspondence: wzsun@ntu.edu.tw
${ }^{6}$ Department of Anesthesiology, National Taiwan University Hospital, Taipei, Taiwan
}

(c) 2010 Wen et al; licensee BioMed Central Ltd. This is an Open Access article distributed under the terms of the Creative Commons Attribution License (http://creativecommons.org/licenses/by/2.0), which permits unrestricted use, distribution, and reproduction in any medium, provided the original work is properly cited. convergent neurons (wide-dynamic-range neurons) at spinal cord or trigeminal nucleus through an inhibitory pathway descending from the lower brainstem [1-4]. Nevertheless, interactions between conditioning stimuli and analgesic responses are largely in unclarity.

DNIC can be triggered by different types of conditioning stimuli, e.g., noxious heat, cold water [5-7], cold presser [8], a brief electrical stimulus [9], and a $\mathrm{CO}_{2}$ laser [10]. However, DNIC effects differ depending on the types of conditioning stimuli, and also on the quality, magnitude, and activated nerve fibers [1,2]. For 
instance, intense electrical stimulation sufficient to activate $A \delta$ and $C$ fibers or to induce pain showed heterotopic analgesia in animals [11-14] and humans [15,16]. In contrast, electroacupuncture (EA), another form of electrical stimulation using a much-lower intensity, also produce remote analgesic effects. It was therefore intriguing to investigate whether analgesic quality is differential between high-intensity (noxious) electrical stimulation and low-intensity EA-like stimulation.

Inflammation also results in pain; however, inflammatory pain-induced DNIC have seldom been studied. A formalin injection in the rat paw results in local inflammation and pain. It was reported that pinch-induced pain at the hindpaw was inhibited by formalin injection in the forepaw by evidence of decrease in Fos expression [17], a pain marker [18-20], suggesting a DNIC effect. However, the presence of DNIC in an inflammatory conditioning may be complicated because studies from monoarthritic animals [21] and rheumatoid arthritic humans [22] indicated that the arthritic duration (acute vs. chronic), pain pattern (evoked vs. constant), and applied pain type (mechanical or thermal) all caused different results in the second pain (test stimulation). Accordingly, the purpose of this study was to investigate underlying differences in DNIC responses to two conditioning stimuli, a supramaximal electrical stimulation and an injection of highdose formalin, applied to the same area. Three sequential steps were undertaken to achieve this aim. First, identify nociceptive qualities of the two conditioning stimuli by behavioral observations and neural activations; second, compare heterotrophic analgesic effects (DNIC) between the two conditioning stimuli by the tail-flick test; and third, with a pharmacological approach, differentiate the recruited descending pathways involved in DNIC responses to the conditioning stimuli.

\section{Methods}

\section{Animal preparation and inhalational anesthetic technique}

Male Sprague Dawley rats (250-350 g, BioLASCO Taiwan, Taipei, Taiwan) were housed in groups of two or three at $23 \pm 1^{\circ} \mathrm{C}$ with a 12 -h dark-light cycle with food and water available ad libitum. Studies were performed under approval of the Animal Care and Use Committee of Shin-Kong Wu Ho-Su Memorial Hospital, and strictly followed Guidelines for the Care and Use of Experimental Animals [23].

All experiments, except that in Methods section 2.2, were conducted under an previously reported anesthetic model [24]. In brief, rats were rapidly anesthetized in an acryl chamber containing halothane-soaked cotton, and then transferred to a transparent tube connected to a breathing circuit pre-filled with $0.75 \%$ halothane in pure oxygen with a flow of $2 \mathrm{~L} / \mathrm{min}$. The halothane concentration was monitored with a gas analyzer. A 10to 15-min "induction period" was necessary to keep animals at a stable anesthetic level [24]. As long as stable tail-flick latencies (TFLs) were obtained, conditioning stimuli were begun, and anesthesia was maintained to the end of the study. Usually, animals recovered to a conscious, freely movable condition in 5 min after anesthesia removal.

\section{Noxious conditioning stimulation}

\subsection{The supramaximal electrical stimulation}

The electrical stimulation was modified from our previous study [24]. One pair of stainless steel needles (30G) was inserted to a depth of $5 \mathrm{~mm}$ in the right acupoint Zusanli (ST36), a point located $5 \mathrm{~mm}$ inferolateral to the right fibular tuberosity and in the upper onethird of the anterior tibial muscle, and a reference point $10 \mathrm{~mm}$ below. Electricity was generated by a Grass S88 stimulator (Astro-med, Grass, Warwick, RI, USA) with two Grass constant current units to deliver the electric current of square pulses at $4 \mathrm{~Hz}$ with a $0.5-\mathrm{ms}$ pulse width. The stimulating current was increased from a level producing local muscle twitching at about 0.3-0.4 $\mathrm{mA}$ (twitch intensity, or abbreviated as TI) to the target intensities within $5 \mathrm{~min}$. Three target intensities were $10 \times \mathrm{TI}$ (3-4 mA; and named as E10), 20×TI (6-8 mA; E20) and a supramaximal intensity (E50), which was as high as the animals could tolerate (usually $50-80 \times \mathrm{TI}$ or $>20 \mathrm{~mA}$ ). Total stimulating period was $30 \mathrm{~min}$. Characteristic rhythmic dorsiflexion of the stimulated hind limb was always seen.

\subsection{Intramuscular (i.m.) formalin injection and weighted pain scores}

To determine which concentration of i.m. formalin would cause a hyperalgesic effect analog to that of an intraplantar (i.pl.) injection, weighted pain scores were used to measure the responsiveness of graded concentrations from $5 \%$ to $20 \%$. First, we tested the appropriate concentration, volume, and depth of formalin required to induce nociceptive behaviors. In conscious rats, 100 $\mu \mathrm{l}$ of $5 \%, 10 \%$, or $20 \%$ formalin, or normal saline (abbreviated as Fm5, Fm10, Fm20, and NS, respectively) was injected into the right Zusanli point. An injection with $50 \mu$ of $2 \%$ formalin ( $\mathrm{Fp}$ ) in the plantar surface of the right hindpaw served as a positive control group. After injection, the rats were transferred to an open iron-wire cage for an 1-h evaluation using a modified weighted pain score method [25]. The scores of an early phase (0-15 $\mathrm{min})$, late phase (20-60 $\mathrm{min})$, and total phase (0-60) were separately calculated for comparison.

To confirm the spread of the injection, $20 \mu \mathrm{l}$ of methylene blue was added to the formalin solution in some rats, and the extent by which the injectate spread in muscles was examined after the animals were sacrificed. 


\subsection{Neuronal activations by conditioning stimuli: Fos immunohistochemistry}

To investigate neuronal activation by the conditioning stimuli, Fos-immunoreactive (Fos-ir) profiles in the lumbar dorsal horns were analyzed. Rats from the Fm20, E20, and E50 groups were sacrificed at $90 \mathrm{~min}$ after the beginning of the conditioning stimulation. Rats were intraperitoneally injected with an overdose of $650 \mathrm{mg} / \mathrm{kg}$ chloral hydrate (Kanto Chemical, Tokyo, Japan) and transcardially perfused with $250 \mathrm{ml}$ of saline followed by $350 \mathrm{ml}$ of $4 \%$ paraformaldehyde in $0.1 \mathrm{M}$ phosphate-buffered saline (PBS, pH 7.4, $4^{\circ} \mathrm{C}$ ). The L2-L5 spinal segments were removed, post-fixed in the same paraformaldehyde solution at $4^{\circ} \mathrm{C}$ for $6 \mathrm{~h}$, and cryoprotected in 30\% sucrose at $4^{\circ}$ $\mathrm{C}$ for $48-72 \mathrm{~h}$. Frozen sections were cut in a cryostat $(30$ $\mu \mathrm{m})$ and collected in PBS as free-floating sections. They were then incubated with primary rabbit polyclonal antiFos antiserum (1: 1500, Santa Cruz Biotechnology, Santa Cruz, CA, USA), and diluted in $0.1 \mathrm{M}$ PBS containing $3 \%$ normal goat serum and $0.3 \%$ Triton $\mathrm{X}-100$ at $4^{\circ} \mathrm{C}$ for $24 \mathrm{~h}$. After washing in PBS, sections were incubated with a biotinylated goat anti-rabbit secondary antibody (1: 200, Vector Laboratories, Burlingame, CA, USA) in PBS for $1 \mathrm{~h}$ at room temperature, and subsequently reacted with the avidin-biotin-peroxidase complex (Elite ABC kit, Vectastain ${ }^{\circledR}$, Vector Laboratories) for $1 \mathrm{~h}$ at room temperature. After rinsing in $0.1 \mathrm{M}$ PBS for $20 \mathrm{~min}$, sections were reacted with a 3,3'-diaminobenzidine tetrahydrochloride solution in PBS containing hydrogen peroxide and nickel (Peroxidase substrate kit, Vector Laboratories) for $6 \mathrm{~min}$. All sections were mounted on gelatin-dubbed slides, air dried, and protected with a coverslip for inspection under a light microscope.

Sections were examined under a Nikon E600 microscope (Tokyo, Japan) using a dark field to determine the segmental levels [26] and a light field for cell counting. The spinal dorsal horn was divided into three regions: (1) the superficial layer (laminae I/II); (2) the nucleus proprius (laminae III/IV); and (3) the deep layer (lamina $\mathrm{V})$. Immunoreactive neurons, which had deep staining distinguishable nuclei from the background, were counted by laminae. For each animal, at least 8-10 sections of each segment were examined, counted, and averaged by segment. Antibody specificity and immunostaining were tested by omission of the primary antibodies. The evaluator who did the counting was blind to the group allocation of the samples.

\section{DNIC effect}

\subsection{Tail flick test as a test stimulus to evaluate analgesic effect}

The DNIC effects were analyzed by tail flick test. With strict control of the ambient temperature at $23^{\circ} \mathrm{C}$, the rat tail was heated at the distal one-third by radiant light from a focused projection bulb in an algesic device (MK-330B, Muromachi Kikai Co. Ltd., Tokyo, Japan). The baseline "tail flick latency" (TFL) was 8-10 s in naive rats, and the tail was passively removed at $20 \mathrm{~s}$, as the "cutoff limit". The "basal latency" was measured after the anesthetic induction period and before the conditioning stimulus, or the time point 0 . The "test latency" at each time point was an average of two successive tests, separated by $2 \mathrm{~min}$, without pause of electrical stimulation. The maximal possible effect (MPE) was calculated as: $\mathrm{MPE} \%=[$ (test latency - basal latency $) /(20$ - basal latency $)] \times 100 \%$.

\subsection{DNIC effects produced by two conditioning stimuli}

Five groups were included: (1) a control group (C) in which rats were inserted with needles but received no electrical stimulation; (2) an E10 group in which rats were given electrical stimulation at $10 \times \mathrm{TI}$; (3) an E20 group in which rats were given electrical stimulation at 20×TI; (4) an E50 group in which rats were given supramaximal electrical stimulation at $50-80 \times \mathrm{TI}$, or as high as the rats could tolerate; and (5) a Fm20 group in which rats were given an i.m. injection of $100 \mu \mathrm{l}$ of $20 \%$ formalin in the right ST36 acupoint. All experiments were conducted under the same conditions of equal anesthetic levels and periods, basal latencies, and TLF time points (Fig. 1). In particular, our anesthetic device allowed three rats to be simultaneously anesthetized, so three groups (electrical, formalin, and control) could be matched under identical conditions and environmental biases would be greatly decreased. Each group contained at least nine rats. Rats were sacrificed at the end of the study for immunostaining.

\section{The mechanistic study of DNIC-mediated analgesia}

To differentiate mechanisms underlying DNIC between the electrical and formalin stimulations, involvement of inhibitory pathways were examined by neurotransmitter antagonists. Two agents were used: naloxone (Genovate Biotech, Hsinchu, Taiwan), an opioid receptor antagonist, and yohimbine (Sigma-Aldrich, St. Louis, MO, USA), a selective $\alpha 2$-adrenoreceptor antagonist. Naloxone was intraperitoneally injected twice, $2 \mathrm{mg} / \mathrm{kg}$ at time point -15 and $1 \mathrm{mg} / \mathrm{kg}$ at time point 30 . Yohimbine was intrathecally (i.t.) injected with a dose of $30 \mu \mathrm{g}$ in $20 \mu \mathrm{l}$ of saline at the time point -15 . The i.t. injection is a single bolus technique at the L5-L6 interspace using a 26-gauge needle and microsyringe (Hamilton, Reno, NV, USA) described elsewhere [27]. Both conditioning stimulations were tested with one of the antagonists and were compared with data of the control groups injected with an equal volume of saline vehicle. At least seven rats were included in each group. 


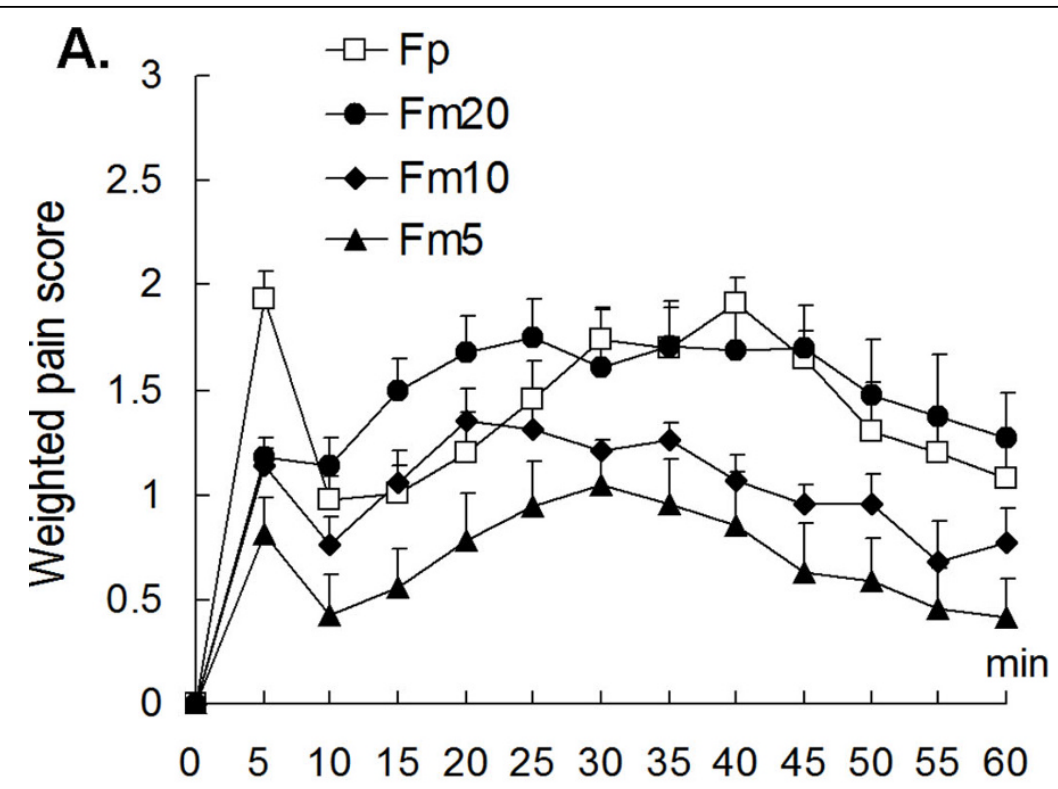

B.

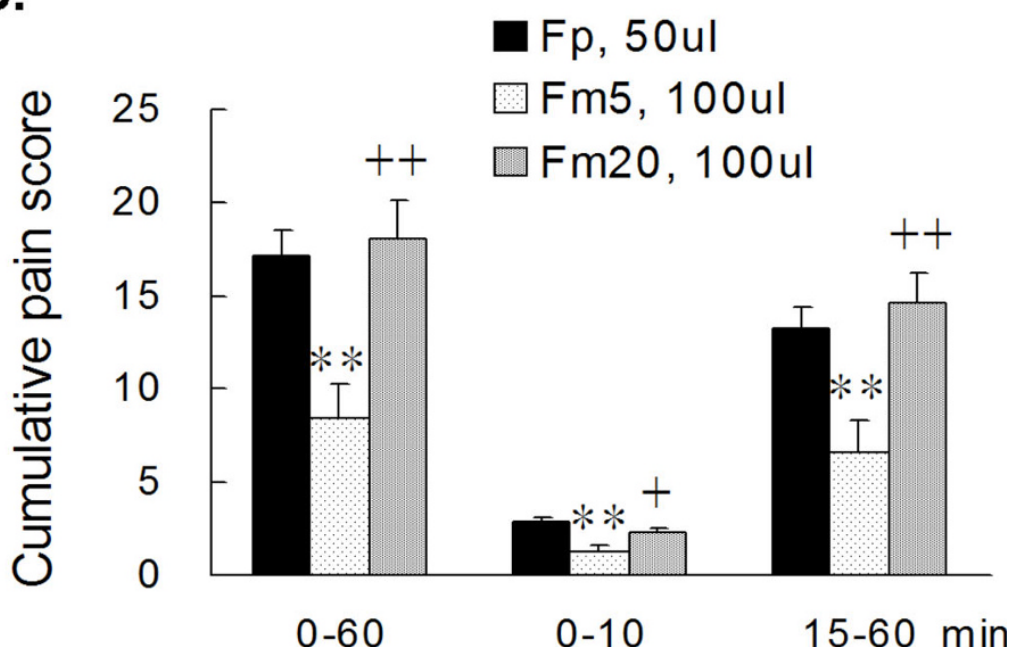

Figure 1 Weighted pain score [24,25] of a formalin injection in the hind limb. (A) An i.m. injection of $100 \mu$ l of 5\% (Fm5), 10\% (Fm10), or 20\% (Fm20) formalin in the anterior tibial muscle induced dose-dependent pain scores and a biphasic pain pattern similar to that of a subcutaneous plantar injection $(2 \%, 50 \mu \mathrm{l}, \mathrm{Fp})$. The Fm20 group had a lower pain score in the early phase and fewer flinch responses for the entire period compared to the Fp group. However, the Fp and Fm20 groups had similar highest pain scores in the late phase. (B) The Fm20 group showed no statistical difference in the cumulative pain score from that of the Fp group, indicating the muscular injection with $100 \mu \mathrm{l}$ of $20 \%$ formalin resulted in a strong noxious reaction. Rat numbers: $\mathrm{Fp}=9$, Fm5 $=7, \mathrm{Fm} 10=6$, Fm20 $=7 .{ }^{* *} p<0.01 \mathrm{vs}^{\mathrm{Fp}}{ }^{{ }^{+}} p<0.05,{ }^{++} p<$ 0.01 vs. Fm5; one-way ANOVA with Bonferroni's post hoc test.

\section{Data analysis}

All quantitative data are expressed as the mean \pm standard error of the mean (SEM). Cumulative values of weighted pain scores at 0-15, 20-60, and 0-60 min, as well as cumulative TFLs over 0-90 min were transformations of the area under curve (AUC). The averaged TFL at each time point, AUCs, and Fos-ir cells were compared with one-way analysis of variance (ANOVA) followed by post hoc Bonferroni's test or Student's $t$-test.
A value of $p<0.05$ was considered statistically significant.

\section{Results}

1. Supramaximal electrical stimulation and i.m. formalin injection induced noxious behaviors

\subsection{Supramaximal electrical stimulation was noxious}

It is apparent that noxious behaviors were shown in the halothane-anesthetized rats subjected to the supramaximal 
electrical stimulation. When the electrical intensity exceeded $50 \times \mathrm{TI}$, a profile of characteristic behaviors including vigorous leg withdrawal, shaking off of the stimulating needles, and/or turn of body in the tube were observed. This finding was consistent with our previous study that most conscious rats cannot tolerate electrical intensities beyond $10 \times \mathrm{TI}$ and exhibit similar behaviors [24]. Though the rats in the current study were anesthetized, it was believed that the E50 stimulation were still noxious enough to induce neuronal reactions.

\subsection{An adequate concentration of injected formalin to induce pain}

Formalin injected into the plantar surface induced stronger pain than injection into the muscles. Biphasic pain pattern, typically seen after i.pl. formalin, was also observed after i.m. injection (Fig. 1), however, some differences in behaviors were shown. Intramuscular formalin induced much fewer flinch activities (pain score $=3$ ) but longer hind paw elevation (pain score $=1-2$ ) compared to those after i.pl. injections.

Dose-dependent hyperalgesic responses were shown in the i.m. formalin groups from concentrations of $5 \%$ to $20 \%$. In the $\mathrm{Fm} 20$ group, pain in the early phase was not so evident, but pain score in the late phase was high because of persistent hind paw elevation. Data analysis showed that the Fm20 and the Fp groups had comparable maximal pain $(1.90 \pm 0.25$ at 35 min in $\mathrm{Fm} 20$ vs. $1.91 \pm 0.11$ at $40 \mathrm{~min}$ in Fp, Fig. 1A) and comparable cumulative scores in the late phase $(14.56 \pm 1.71$ for the Fm20 vs. $13.23 \pm 1.12$ for the Fp, $p=1.00$ ) (Fig 1B). In the early phase, the Fm 20 group had a lower score than the Fp group but the difference was insignificant $(p=0.50)$. Therefore, an i.m. injection of $100 \mu \mathrm{l}$ of $20 \%$ formalin was proved to be noxious and this dose was chosen as a conditioning stimulus.

The spread of methylene blue was examined in four rats. Dense deep-blue staining was confined to the deep layer of the anterior tibial muscle and was scarcely dispersed through the interosseous membrane. No blue staining was found in the posterior calf muscles.

\section{Supramaximal electrical stimulation and i.m. formalin both induced a significant increase in Fos expression in the spinal dorsal horns}

Whether the two conditioning stimuli produced different neuronal responses were examined with a profile of Fos-ir expression in the spinal dorsal horn. As shown in Fig. 2, rats in the control and E20 groups (low-intense stimulation) exhibited very few Fos-ir profiles from the L2 to L5 dorsal horns (Fig. 2A, B, G, H); however, the E50 and Fm20 groups showed marked Fos expressions in the superficial laminae (Fig. 2C, E), which were significantly higher than those of the control $(p<0.01)$ and E20 groups ( $p<0.05$ or 0.01 , Fig. 3 ) at L2-L5 segments
(Fig. 3). In comparison, Fos expression of the E50 group was densely distributed in the medial half of the superficial laminae of the L2-L3 segments, whereas Fos in the Fm20 group was loosely scattered in the superficial laminae of the same segments. In the lower L4-5 segments, Fos-ir cells of both the E50 and Fm20 groups were evenly expressed in superficial layers (Fig. 2D, F). The activated patterns of postsynaptic neurons were thus shown differently between the two conditioning stimulations.

\section{Both noxious stimulations produced DNIC}

\subsection{Electrical stimulation prolonged TFLs in an intensity- dependent manner}

Under constant anesthesia, the control group maintained stable TFLs for $90 \mathrm{~min}$, and graded electrical stimulation produced intensity-dependent suppression on tail-flick withdrawals during and after the stimulation period (Fig. 4A, B). E10 stimulation, using an EA-like intensity, mildly prolonged the TFL from time 0 to 40 , and showed an after-effect during time 80 to 90 . The maximal MPE of the E10 at time 20 was significantly higher than that of the control $(p<0.001)$. The supramaximal E50 stimulation produced strong analgesia for over $90 \%$ withdrawal inhibition at time $20-30$, followed by a prolonged after-effect. Nevertheless, the E50 rat did not show traumatic signs, such as licking, elevating, limping, or local tissue inflammation at $1 \mathrm{wk}$ of followup.

\subsection{Formalin injection produced a different pattern of tail} flick depression

Noxious formalin $(20 \%, 100 \mu \mathrm{l})$ suppressed tail withdrawal with a pattern differed from that of E50 stimulation. Immediate and short-lived analgesia occurred in the first $10 \mathrm{~min}$ after the injection, followed by increasing suppression of the tail-flick response (Fig. 4C). Obviously, the DNIC effect was correlated with an inflammatory process of the tibial muscle. This formalin-induced ongoing pain differed from the E50-evoked short-term pain, because the latter depends on the existence of electrical stimulation. Even though the current data revealed that both stimuli may have distinct DNIC patterns, there were no differences in the cumulative pain scores (Fig. 4D).

\section{DNIC induced by supramaximal electrical and noxious formalin stimulation were mediated by different inhibitory pathways}

Interestingly, the naloxone injection did not significantly reverse E50-induced DNIC in tail-flick responses. No statistical difference was found between the E50 and E50 + Nal groups, regardless of the time-to-time comparison or cumulative analgesic calculation (E50 vs. E50+Nal, $p>0.05$ ) (Fig. 5A, B). Meanwhile, it was shown that 

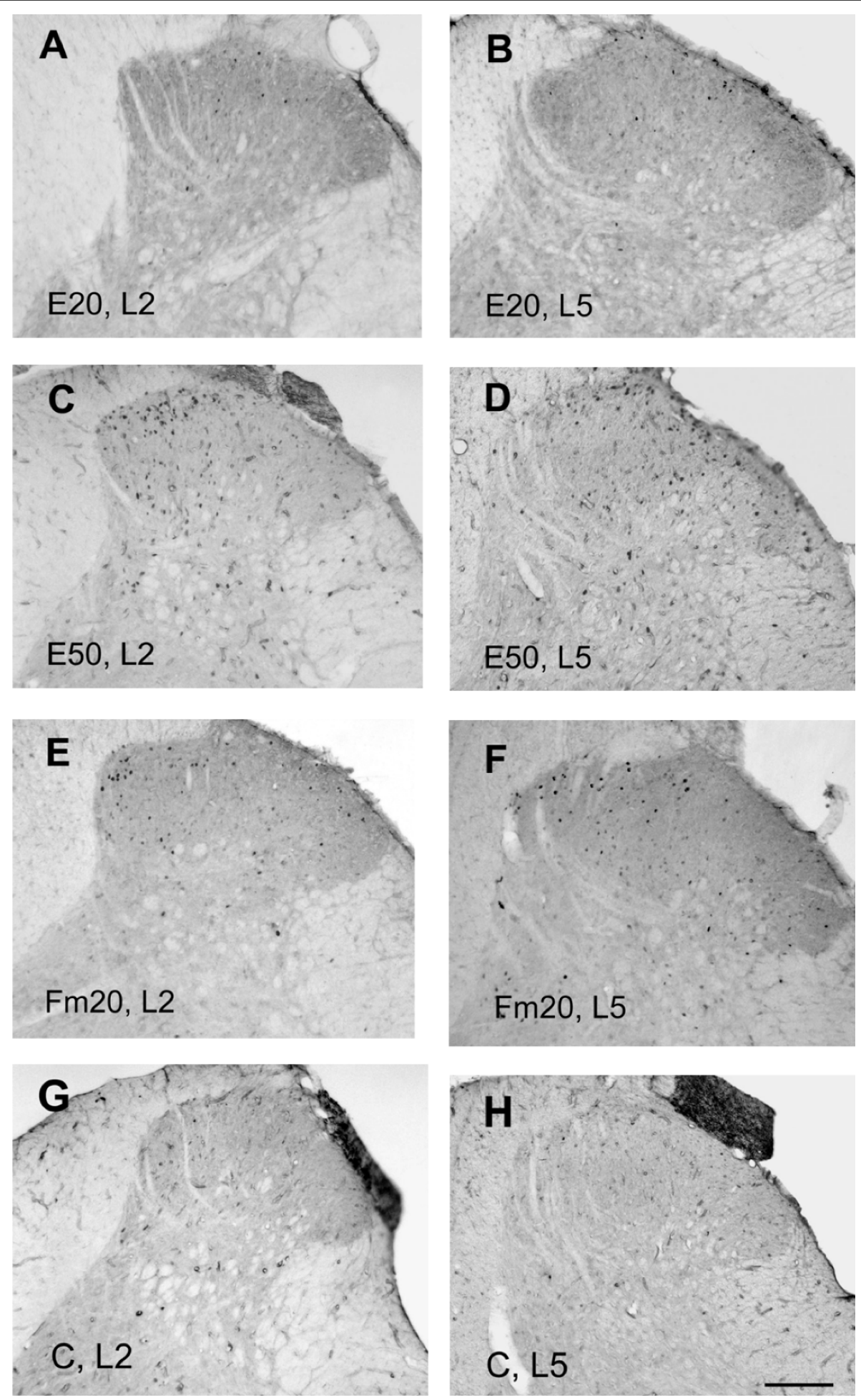

Figure 2 Rostrocaudal distribution of Fos-immunoreactive (Fos-ir) neurons after two conditioning noxious stimulations. Fos-ir neurons were identified at the $L 2(A, C, E, G)$ and $L 5(B, D, F, H)$ spinal dorsal horn at 90 min after the $E 20$ and Fm20 stimulations. The groups shown in the figure are: the $E 20(A, B), E 50(C, D), F m 20(E, F)$, and control groups $(G, H)$. Fos-ir neurons were few in the control and E20 rats at all segments (A, B, G, H). Formalin (Fm20) and supramaximal electrical (E50) stimulation induced Fos expression in L2 and L5 superficial laminae (C-F). In comparison, E50-induced Fos-ir neurons were densely distributed at the medial one-third of the L2 superficial dorsal horn (C), whereas Fm20-induced expression was relatively loosely distributed in the superficial dorsal horns (E). Scale bar $=100 \mu \mathrm{m}$. 
A
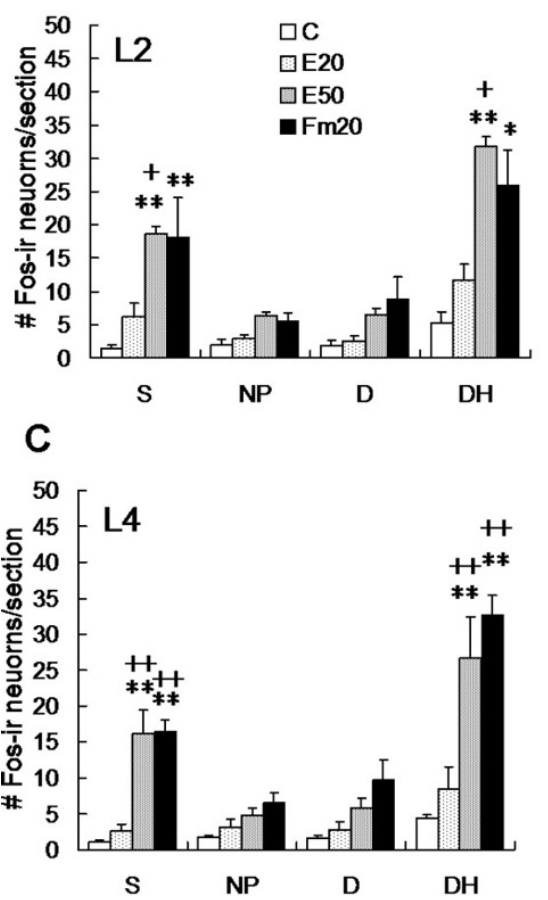

B
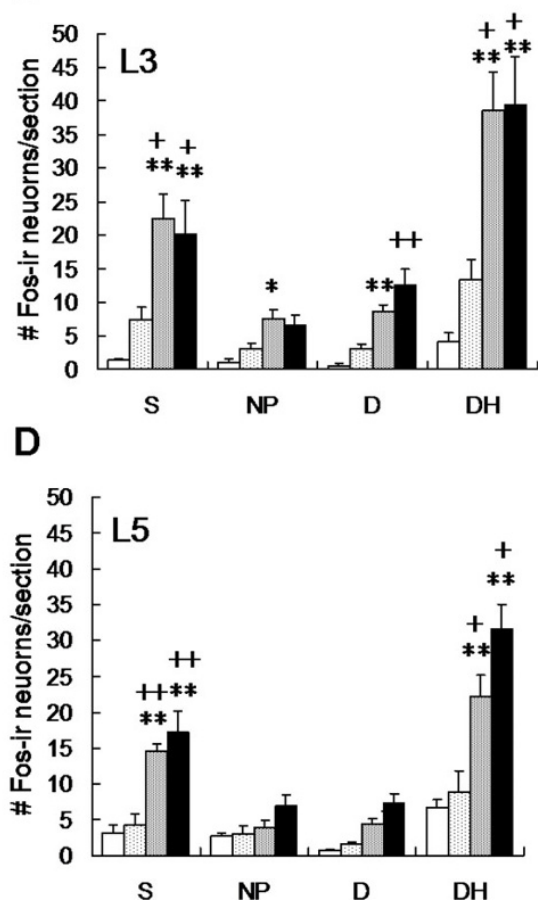

Figure 3 Numerical analysis of Fos-ir neurons at the side ipsilateral to the conditioning stimulus. Fos-labeled neurons were significantly higher in the Fm20 and E50 groups than in the control and E20 groups regardless of the spinal segment. No significant difference was found between the C and E20 groups, or between the Fm20 and E50 groups for all segments and laminae. Topographically, E50-induced Fos expression was higher in the higher segments (L2 and L3) than in the lower lumbar segments (L4 and L5). S, superficial laminae I/Il; NP, nucleus proprius, laminae III/IV; D, deep laminae V; DH, dorsal horn. Rat numbers: $\mathrm{C}=6, \mathrm{E} 20=6, \mathrm{E} 50=5, \mathrm{Fm} 20=6 .{ }^{*} p<0.05,{ }^{* *} p<0.01$ vs. $\mathrm{C} ;{ }^{+} p<$ $0.05,{ }^{++} p<0.01$ vs. E20; one-way ANOVA with Bonferroni's post hoc test.

naloxone per se did not affect basal TFLs in the control. On the other hand, naloxone evidently reversed Fm20induced DNIC, and antagonism was shown in the early (time 10) and late (time points 60, 70, and 90) periods (all $p<0.05$, Fig. 5C). The Fm $20+\mathrm{Nal}$ group had a significantly lower area under the curve (AUC) than the Fm20 group by $57 \%(p<0.05)$ (Fig. 5D). The results showed that noxious formalin, but not E50 stimulation, produced an opioid-dependent DNIC.

In contrast, the selective $\alpha 2$ receptor antagonist, yohimbine, showed a different action. Intrathecal yohimbine did not affect the basal TFLs in the control; however, DNIC of both conditioning nociception were significantly attenuated by i.t. administration of $30 \mu \mathrm{g}$ yohimbine (Fig. 6). In both the E50 and Fm20 groups, yohimbine reversed DNIC for long-lasting periods (Fig. 6A, C). The analgesic summation (AUC) demonstrated a strong DNIC reversion of over $60 \%$ in both groups (In E50, from $45.35 \pm 6.27$ to $17.99 \pm 7.28$, $p<0.05$; in Fm20, from $47.06 \pm 4.15$ to $10.99 \pm 4.06$, $p<0.001)$. The results proved that the $\alpha 2$-adrenergic pathway is involved in DNIC produced by either noxious electrical or formalin stimulation.

\section{Discussion}

Our study on DNIC from two different types of conditioning stimuli, the supramaximal electrical stimulation and high-concentration formalin injection, reveals several findings. First, under a minimal-stress anesthetic condition, the supramaximal electrical stimulation, usually $>20 \mathrm{~mA}$, induced much-stronger suppression of the tail withdrawal reflex than a low-intensity EA-like stimulation; second, formalin injection-induced muscular pain also elicited DNIC; third, noxious electrical and formalin stimulation induced Fos expression with a distinct topographical distribution in the spinal dorsal horns; and fourth, most importantly, the two conditioning stimuli triggered distinct underlying inhibitory pathways.

\section{The DNIC behaviors differed between the two conditioning stimuli}

DNIC has been suggested to be dependent on the conditioning stimuli of different qualities, noxious intensities, durations, and locations [9,28-31]. We found an intensity-dependent inhibition of the tail flick reflex by graded conditioning electrical stimulations in this and a 
A.

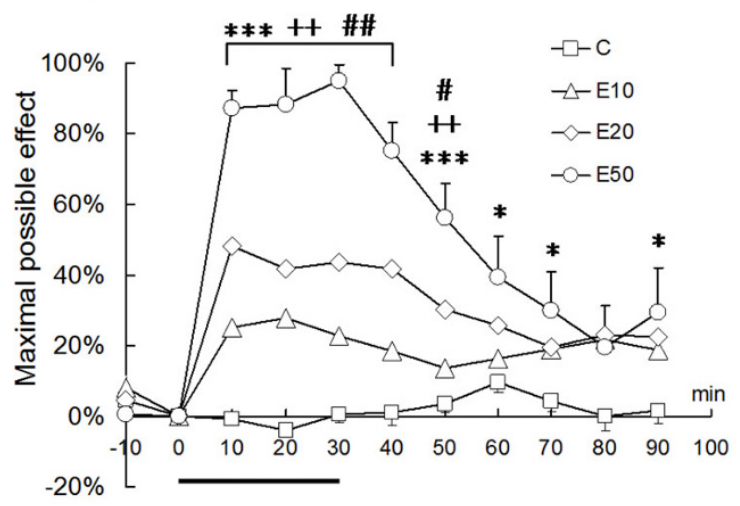

C.

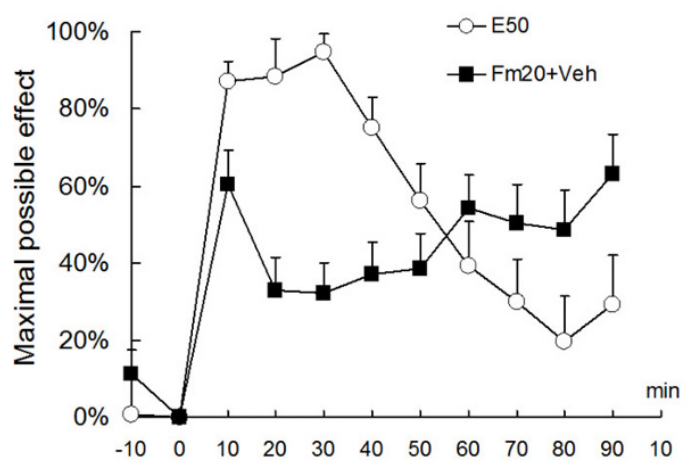

B.

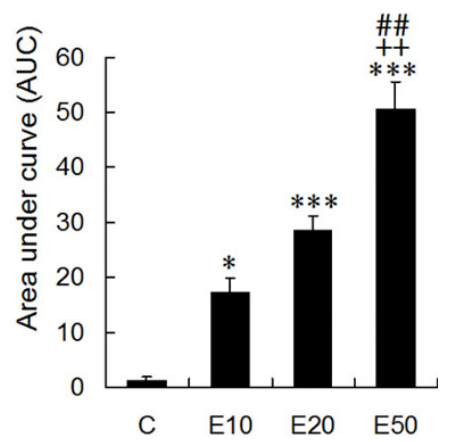

D.

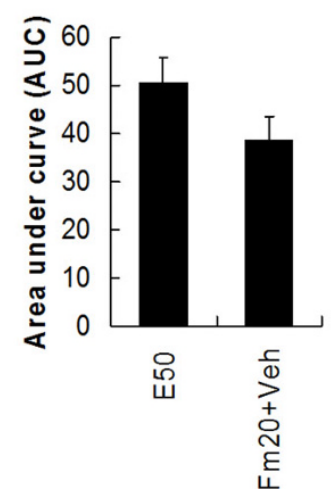

Figure 4 Effect of noxious electrical and formalin stimulation-induced DNIC on the tail-flick latency. (A, B) Halothane-anaesthetized rats were grouped into sham needles (C), E10 (10x twitch intensity), E20, and E50 (> 50x) electrical stimulation of the right ST36 acupoint. Changes in tail-flick latencies were compared by the "maximal possible effect". Control rats exhibited consistent tail-flick latencies without anesthetic influence. The electrical stimuli produced intensity-dependent analgesia on the tail reflex and showed maximal DNIC effects within the stimulation period. Notably, E50 elicited a strong and long analgesic effect (B). (C, D) A high-dose formalin injection (Fm20) caused a distinct DNIC pattern from E50 stimulation in tail-flick suppression. However, total pain summation (area under the curve) indicated no statistical difference between E50 and Fm20 (D). The horizontal thick bar indicates the electrical stimulating period. Rat numbers: $C=11, E 10=10$, $\mathrm{E} 20=11, \mathrm{E} 50=9, \mathrm{Fm} 20=10 .{ }^{*} p<0.05,{ }^{* * *} p<0.001$ vs. $\mathrm{C}_{i}{ }^{++} p<0.01,{ }^{+++} p<0.001$ vs. E10; ${ }^{\#} p<0.05,{ }^{\# \# \#} p<0.001$ vs. E20; one-way ANOVA with Bonferroni's post hoc test.

previous study [24]. In addition, we also showed different DNIC responses to various conditioning stimuli. The maximal DNIC effect in the E50 group appeared at the end of the electrical stimulus, whereas in the formalin group, the effect exhibited two peaks, one at the beginning and the other at the end of observation. The results apparently reflect a correlation between DNIC and noxious levels of the conditioning stimuli.

The activated peripheral nociceptors and projecting neurons by electricity and formalin may differ, which can partially explain the variations in DNIC. It is possible that E50 excites certain groups of mechano-receptors concentric to the electrical field, while injected formalin might diffuse to a broader region in the muscles and sensitize different groups of mechano- and chemo-receptors. In the meanwhile, the variation in the spinal Fos distribution provides additional evidence that post-synaptic neurons were differentially activated by both stimuli. Although Fos-expressing mapping is insufficient to justify the nociceptive quality, our immunostaining data are still informative at disclosing variations in the activation of CNS pathways.

\section{Supramaximal electrical stimulation-induced DNIC is} naloxone irreversible, but yohimbine reversible

The supramaximal electrical stimulation in this study is an extrapolated example of EA, an intentional design which can be compared to our previous study [24]. The endogenous opioid system is a pivotal mechanism in EA analgesia [32-34] and also contributes to DNIC [35,36]. 


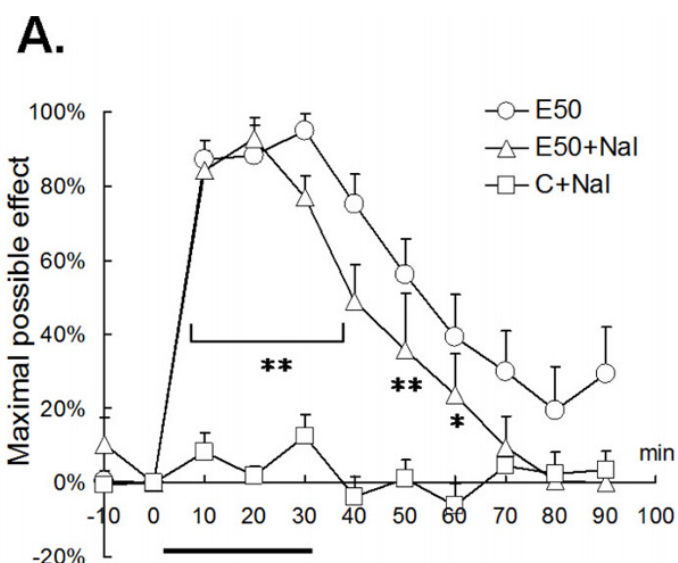

C.

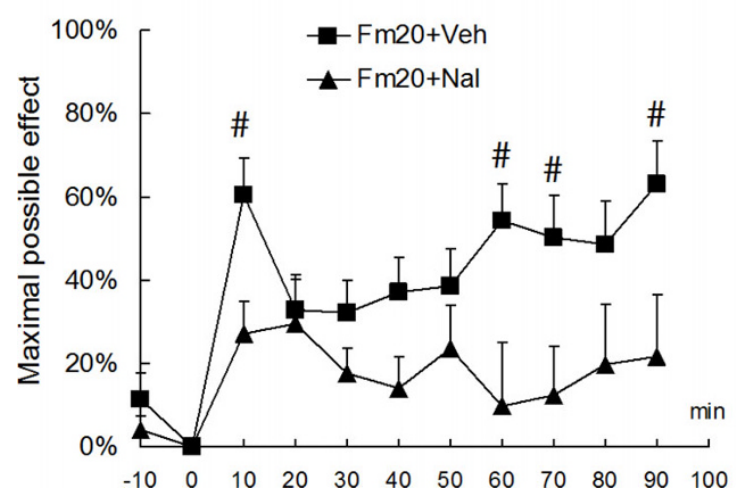

B.

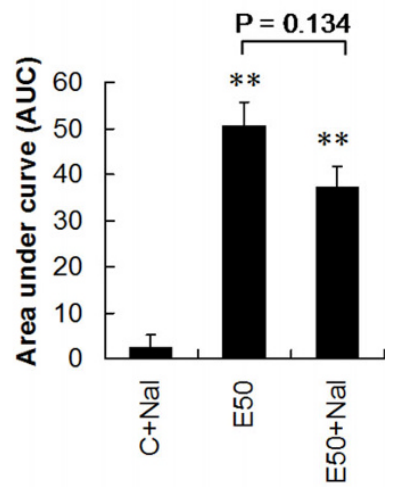

D.

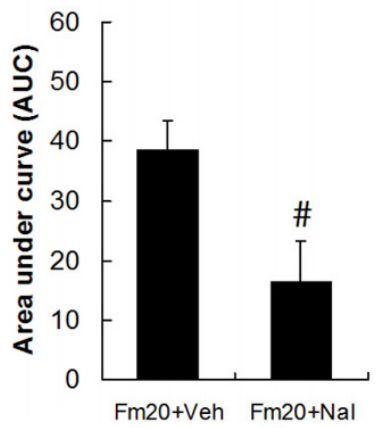

Figure 5 Contribution of the opioidergic pathway to DNIC. The opioid receptor was antagonized by a $2 \mathrm{mg} / \mathrm{kg}$ intraperitoneal naloxone injection at time point 15 and $1 \mathrm{mg} / \mathrm{kg}$ at time point 30. Naloxone itself did not alter the tail-flick latency in the control group (A, the C+Nal line). (A, B) The E50-induced DNIC was not reversed by naloxone administration, whereas the Fm20-induced DNIC was reversed in both the early and late phases by time point-to-point comparisons (C) or by total pain summation (D). Veh, saline; Nal, naloxone. The horizontal thick bar indicates the electrical stimulating period. Rat numbers: $\mathrm{E} 50+\mathrm{Veh}=9, \mathrm{E} 50+\mathrm{NAL}=7, \mathrm{C}+\mathrm{NAL}=9, \mathrm{Fm} 20+\mathrm{Veh}=10, \mathrm{Fm} 20+\mathrm{Nal}=9 .{ }^{* *} p<0.01$, *** $p<0.001$ vs. C+Nal; ${ }^{\#} p<0.05$ vs. Fm20+Nal; one-way ANOVA with Bonferroni's post hoc test.

However, whether DNIC is involved in EA analgesia is quite controversial $[16,31,37,38]$. Moreover, it was suggested that different mechanisms could be triggered to suppress evoked potentials and tooth pain when the intensities increased from just activating large afferent A fibers to sufficiently recruiting $C$ fibers $[9,29]$.

In contrast to the concept that naloxone inhibited EA analgesia $[24,39,40]$, we did not find a naloxone-reversible DNIC in the supramaximal E50 group. Since the supramaximal electrical stimulus activated broader neural circuits (all types of sensory afferents) and brain areas than did the lower-intense stimulations like EA (which only activated $A \beta$ and $A \delta$ fibers), it is presumed that as the electrical intensity increases from low (maybe no pain or only minimal pain) to high (strong pain), there is a shift in the triggered mechanisms in the central nervous system. Despite there were arguments whether endogenous opioids participating in acupuncture in rats [41-44], rabbits [45], and humans [46,47], our data in the first instance suggest that it is better to have a clear demarcation of the electrical intensity by which the underlying mechanisms of DNIC analgesia and acupuncture analgesia may differ.

On the other hand, yohimbine could reverse, though partially, E50-induced DNIC analgesia. In another study, when rats received $10 \times \mathrm{EA}$, the analgesia on an ankle sprain was reversed by yohimbine and phentolamine, a non-selective $\alpha$ antagonist, but not by terazosin, an $\alpha 1$ adrenergic antagonist [48]. Therefore, it was shown that descending analgesia of electrical stimulation is comprised, at least partly, of $\alpha 2$-adrenoceptor-mediated inhibition regardless of the stimulating intensity at a noxious or innocuous level. Taken together, electricitytriggered analgesia is classified into at least two 


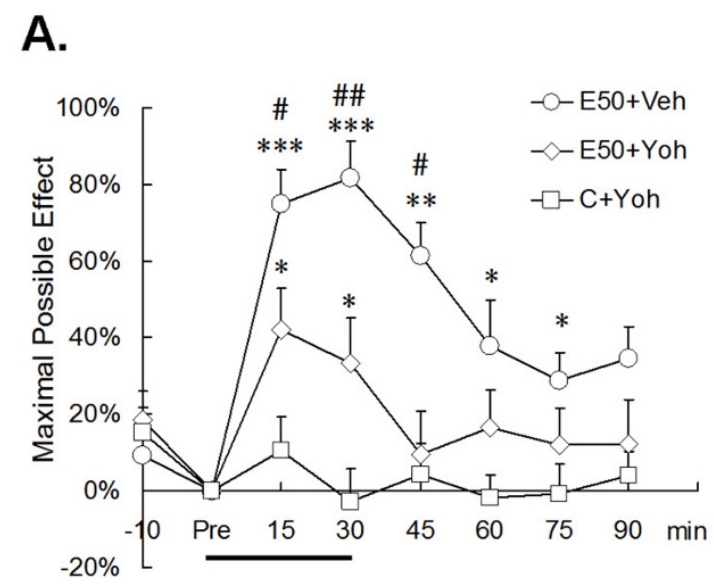

C.

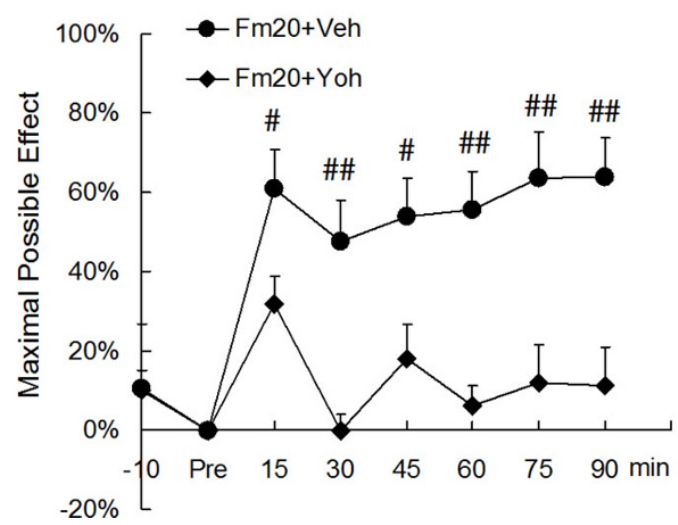

B.

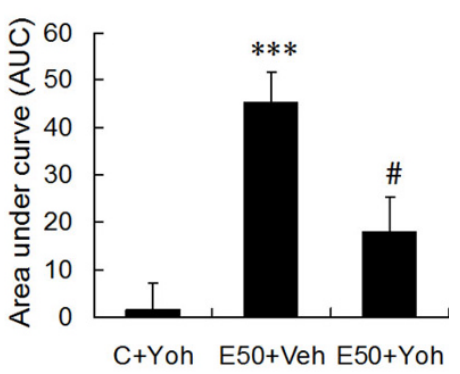

D.

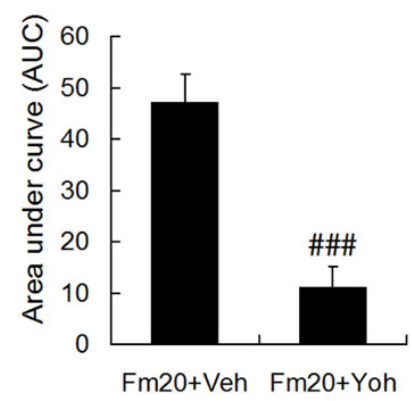

Figure 6 Contribution of the $\boldsymbol{\alpha} \mathbf{2}$-adrenergic pathway to DNIC. The $\alpha 2$-adrenergic receptor was antagonized by an intrathecal yohimbine injection of $30 \mu \mathrm{g}$ in $20 \mu \mathrm{l}$ of saline, $15 \mathrm{~min}$ before the conditioning stimulus. Yohimbine did not alter the tail-flick latency in the control group ( $A, C+$ Yoh line). Unlike naloxone, yohimbine had the ability to reverse the DNIC effect produced by both E50 (A, B) and Fm20 (C, D). Veh, saline; Yoh, yohimbine. Rat numbers: E50+Veh =9, E50+Yoh = 10, C+Yoh = 10, Fm20+Veh =9, Fm20+Yoh = 9. ${ }^{*} p<0.05,{ }^{* *} p<0.01,{ }^{* * *} p<0.001$ vs. C+Yoh; ${ }^{\#} p<0.05,{ }^{\# \#} p<0.01$ vs. E50+Yoh (A, B) or vs. Fm20+Veh (C, D), respectively; one-way ANOVA with Bonferroni's post hoc test.

mechanisms: an opioid-related mechanism predominating at low intensities and a $\alpha 2$ adrenergic system covering a much-wider range of intensities (Fig. 7).

\section{Noxious formalin-induced DNIC is both naloxone and yohimbine reversible}

We found that high-dose formalin induces local pain and triggers DNIC which is reversed by naloxone. The formalin injection caused local inflammation and tissue injuries including muscles, fascia, vessels, and/or nerves. In addition to direct sensitization of the central endogenous opioid system, the inflammation activated release of proinflammatory cytokines and chemokines, and enhanced the production of leukocyte-derived opioid peptides $[49,50]$. For instance, Freund's adjuvantinduced inflammation showed an peripheral action of leukocyte-derived $\beta$-endorphin, met-enkephalin, and dynorphin on the $\mu, \delta$, and $\kappa$ receptors, respectively, and opioid-mediated antinociception [51]. Therefore, the naloxone-reversible component in formalin-induced DNIC could include both central and peripheral opioid actions because intraperitoneal injection of naloxone could be systemically absorbed. By no means, it is demonstrated that formalin-induced DNIC consists of opioid and non-opioid mechanisms, and the latter may be inflammation-independent as in noxious electricityinduced DNIC and $\alpha 2$-recptor mediated (Fig. 7).

Descending inhibitory modulation mediated through spinal $\alpha 2$ receptor activation has been largely reported. Electrical stimulating peripheral $\mathrm{A} \delta$ and $\mathrm{C}$ fibers [52,53] or central noradrenergic cells [54] were found to trigger the descending adrenergic system and release norepinephrine in the spinal cord. Accumulating studies demonstrated that spinal norepinephrine administration [55-58] induced powerful antinociception or inhibited the amplitude of monosynaptically evoked A delta-fiber 


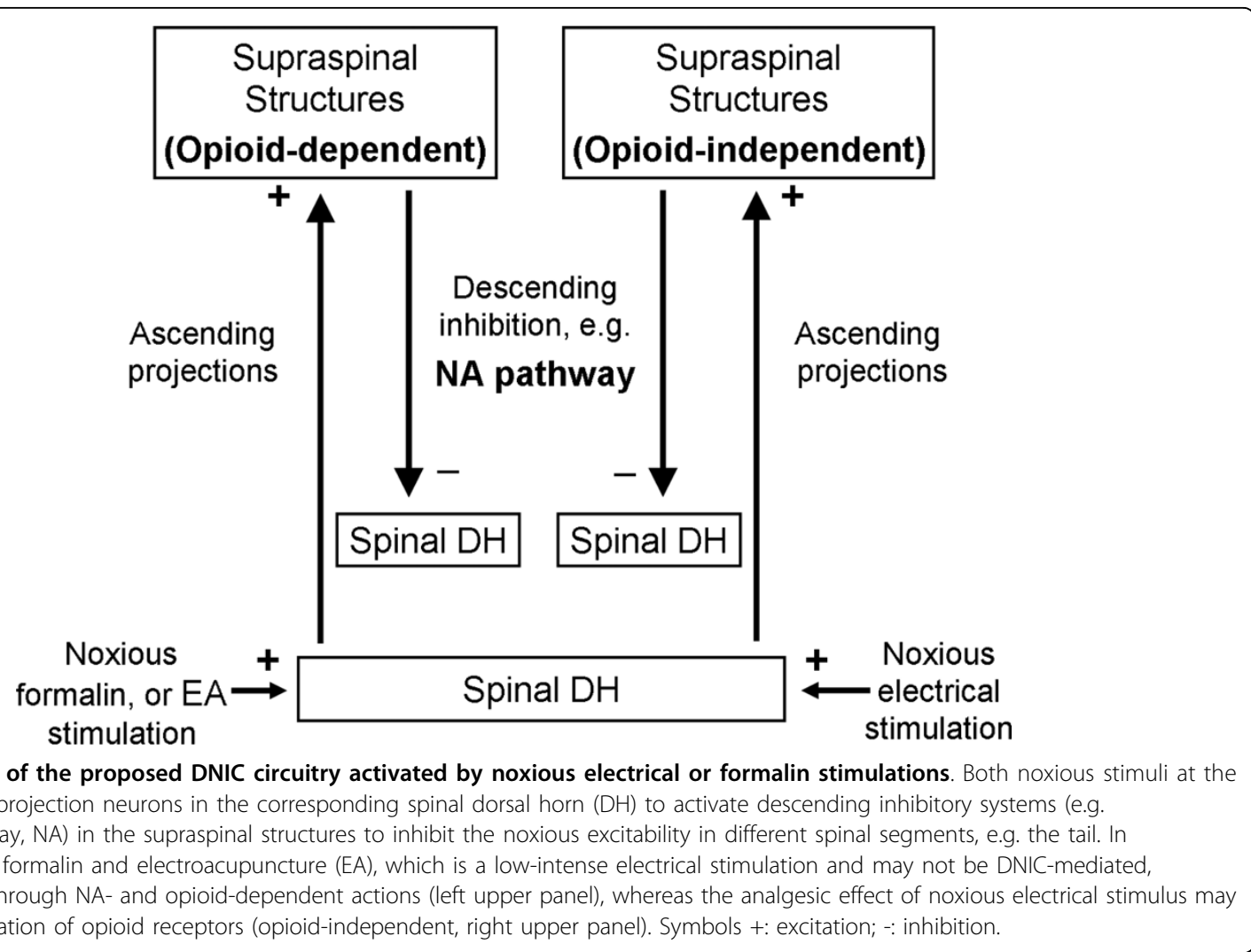

and C-fiber excitatory postsynaptic currents in a whole cell patch clamp technique [59]. However, spinal and pontine $\alpha 2$-adrenoceptors have opposite effects on painrelated behavior in neuropathic animals [60]. The current study provides additional evidence that a formalin injection stimulates yohimbine-reversible heterotopic analgesia. Conclusively, it is suggested that activation of the descending $\alpha 2$-adrenergic pathway may be a universal mechanism in DNIC effect through a variety of conditioning stimuli $[56,61,62]$.

\section{Formalin-induced noxious inhibition or facilitation}

Central nociceptive activation following peripheral inflammation should be meticulously interpreted based on various conditions. Contradictory results may be obtained because the opposing forces of descending modulations (facilitation $v s$. inhibition) can be simultaneously activated $[3,63]$. This study revealed that high-dose formalin induced DNIC analgesia. However, nociceptive hypersensitivity with a receptive field expansion is another probable consequence of prolonged inflammation due to long-term potentiation [64]. In rodents with acute monoarthritis (< $48 \mathrm{~h}$ ), inhibition of trigeminal convergent neurons was produced by mechanical or thermal stimulation of the arthritic joint, whereas chronic arthritis of over $3 \mathrm{wk}$ did not show a DNIC effect [21]. A human study revealed no difference in DNIC responses among patients with rheumatoid arthritis of over 5 years, $<1$ year, and healthy controls. However, those 5-yr arthritic patients showed more pressure allodynia on the non-painful thighs than did 1-yr patients, indicating higher sensitization, but not inhibition, of somatosensory functions [22]. In these studies, pain duration played a critical role in determining the balance skewed to either side of two opposing forces. Unfortunately, many other factors, such as noxious qualities (e.g., inflammatory $v s$. neuropathic), can bias this tug-of-war. Therefore, more studies are necessary before introducing DNIC in clinical treatment.

\section{Clinical implications}

The current DNIC study throws some light on EA application. For a long time, the acupuncture "dose" was efficacy relevant but remains a vague idea. Because acupuncture stimulating strength is largely empirical and the analgesic effect is also affected by psychological stressors such as nervousness, anxiety, and fear [34,65-68], it is difficult to predict the effectiveness of acupuncture in awake humans.

We found that stronger EA stimulation was more effective. Since EA analgesia is weak, equivalent to a morphine dose of 0.5 [69] to $2 \mathrm{mg} / \mathrm{kg}$ [24], increasing the electrical intensity can possibly produce a higher 
effect. For this reason, patients under general anesthesia are a suitable group of subjects to apply strong EA stimulation. This assumption agrees with our clinical study that preoperative high-frequency EA, which accumulated greater energy output, resulted in less morphine consumption than did the lower-frequency EA in the postoperative period [70]. Certainly, the highest limit of electrical power without causing tissue damage should be determined beforehand.

\section{Conclusions}

DNIC is a well-known physiological phenomenon; however, its clinical value and application are unclear. This animal study provides information of differential consequences and mechanisms produced by two qualities of conditioning stimuli, supramaximal electrical stimulation and a noxious formalin injection. Clinical implications of the two noxious stimuli are respectively discussed on the basis of inflammatory states and perioperative analgesia. We suggest that greater understanding of DNIC analgesia, by structuring the complex descending circuitry with specific mechanisms under different conditioning, will help turn this theory into a useful clinical pain control application.

\section{Acknowledgements}

The authors thank Yi-Hao Chang for technical assistance. This study was supported by grants NSC90-2314-B-341-005 and NSC97-2314-B-341-002 in part (to YRW) and NSC89-2314-B002-302 (to WZS) from the National Science Council of Taiwan and grant 8302-90-0208-01 (to YRW) from Shin-Kong Wu Ho-Su Memorial Hospital.

\section{Author details \\ ${ }^{1}$ Graduate Institute of Clinical Medicine, College of Medicine, Taipei Medical University, Taipei, Taiwan. ${ }^{2}$ School of Medicine, College of Medicine, Taipei Medical University, Taipei, Taiwan. ${ }^{3}$ Department of Anesthesiology, Shin-Kong Wu Ho-Su Memorial Hospital, Taipei, Taiwan. ${ }^{4}$ School of Medicine, Fu Jen Catholic University, Taipei County, Taiwan. ${ }^{5}$ Graduate Institute of Acupuncture Science, China Medical University, Taichung, Taiwan. ${ }^{6}$ Department of Anesthesiology, National Taiwan University Hospital, Taipei, Taiwan.}

\section{Authors' contributions}

YRW and WZS conceived of the study, designed and performed the experiments, analyzed the data, and wrote the manuscript. CCW participated in analyzing and revised the manuscript. GCY and SFH helped to design and coordinate the study, and participated in drafting the manuscript. YLL and $\mathrm{YJH}$ carried out the behavioral observations of the experiments. All authors read and approved the final manuscript.

\section{Competing interests}

The authors declare that they have no competing interests.

Received: 21 November 2009 Accepted: 19 March 2010 Published: 19 March 2010

\section{References}

1. Le Bars D, Dickenson AH, Besson JM: Diffuse noxious inhibitory controls (DNIC). I. Effects on dorsal horn convergent neurones in the rat. Pain 1979, 6:283-304.
2. Le Bars D, Dickenson AH, Besson JM: Diffuse noxious inhibitory controls (DNIC). II. Lack of effect on non-convergent neurones, supraspinal involvement and theoretical implications. Pain 1979, 6:305-327.

3. Le Bars D: The whole body receptive field of dorsal horn multireceptive neurones. Brain Res Brain Res Rev 2002, 40:29-44.

4. Hu JW: Response properties of nociceptive and non-nociceptive neurons in the rat's trigeminal subnucleus caudalis (medullary dorsal horn) related to cutaneous and deep craniofacial afferent stimulation and modulation by diffuse noxious inhibitory controls. Pain 1990, 41:331-345.

5. Price DD, McHaffie JG: Effects of heterotopic conditioning stimuli on first and second pain: a psychophysical evaluation in humans. Pain 1988, 34:245-252.

6. Kakigi $R$, Watanabe $S$ : Pain relief by various kinds of interference stimulation applied to the peripheral skin in humans: pain-related brain potentials following CO2 laser stimulation. J Peripher Nerv Syst 1996, 1:189-198.

7. Watanabe S, Kakigi R, Hoshiyama M, Kitamura Y, Koyama S, Shimojo M: Effects of noxious cooling of the skin on pain perception in man. J Neurol Sci 1996, 135:68-73.

8. Johannesson U, de Boussard CN, Brodda Jansen G, Bohm-Starke N: Evidence of diffuse noxious inhibitory controls (DNIC) elicited by cold noxious stimulation in patients with provoked vestibulodynia. Pain 2007, 130:31-39.

9. Motohashi K, Umino M: Heterotopic painful stimulation decreases the late component of somatosensory evoked potentials induced by electrical tooth stimulation. Brain Res Cogn Brain Res 2001, 11:39-46.

10. Oono Y, Fujii K, Motohashi K, Umino M: Diffuse noxious inhibitory controls triggered by heterotopic $\mathrm{CO} 2$ laser conditioning stimulation decreased the SEP amplitudes induced by electrical tooth stimulation with different intensity at an equally inhibitory rate. Pain 2008, 136:356-365.

11. Morton CR, Du HJ, Xiao HM, Maisch B, Zimmermann M: Inhibition of nociceptive responses of lumbar dorsal horn neurones by remote noxious afferent stimulation in the cat. Pain 1988, 34:75-83.

12. Romita W, Henry JL: Intense peripheral electrical stimulation differentially inhibits tail vs. limb withdrawal reflexes in the rat. Brain Res 1996, 720:45-53.

13. Romita W, Suk A, Henry JL: Parametric studies on electroacupuncture-like stimulation in a rat model: effects of intensity, frequency, and duration of stimulation on evoked antinociception. Brain Res Bull 1997, 42:289-296.

14. Danziger N, Gautron M, Le Bars D, Bouhassira D: Activation of diffuse noxious inhibitory controls (DNIC) in rats with an experimental peripheral mononeuropathy. Pain 2001, 91:287-296.

15. Roby-Brami A, Bussel B, Willer JC, Le Bars D: An electrophysiological investigation into the pain-relieving effects of heterotopic nociceptive stimuli. Probable involvement of a supraspinal loop. Brain 1987, 110(Pt 6):1497-1508.

16. Xu WD, Zhu B, Rong PJ, Bei H, Gao XY, Li YQ: The pain-relieving effects induced by electroacupuncture with different intensities at homotopic and heterotopic acupoints in humans. Am J Chin Med 2003, 31:791-802.

17. Boucher $T$, Jennings $E$, Fitzgerald $M$ : The onset of diffuse noxious inhibitory controls in postnatal rat pups: a C-Fos study. Neuroscience Letters 1998, 257:9-12.

18. Harris JA: Using c-fos as a neural marker of pain. Brain Res Bull 1998, 45:1-8.

19. Hunt SP, Pini A, Evan G: Induction of c-fos-like protein in spinal cord neurons following sensory stimulation. Nature 1987, 328:632-634.

20. Presley RW, Menetrey D, Levine JD, Basbaum Al: Systemic morphine suppresses noxious stimulus-evoked Fos protein-like immunoreactivity in the rat spinal cord. J Neurosci 1990, 10:323-335.

21. Danziger N, Weil-Fugazza J, Le Bars D, Bouhassira D: Alteration of descending modulation of nociception during the course of monoarthritis in the rat. J Neurosci 1999, 19:2394-2400.

22. Leffler AS, Kosek E, Lerndal T, Nordmark B, Hansson P: Somatosensory perception and function of diffuse noxious inhibitory controls (DNIC) in patients suffering from rheumatoid arthritis. Eur J Pain 2002, 6:161-176.

23. Zimmermann M: Ethical guidelines for investigations of experimental pain in conscious animals. Pain 1983, 16:109-110.

24. Wen YR, Yeh GC, Shyu BC, Ling QD, Wang KC, Chen TL, Sun WZ: A minimal stress model for the assessment of electroacupuncture analgesia in rats under halothane. Eur J Pain 2007, 11:733-742. 
25. Dubuisson D, Dennis SG: The formalin test: a quantitative study of the analgesic effects of morphine, meperidine, and brain stem stimulation in rats and cats. Pain 1977, 4:161-174.

26. Molander C, Xu Q, Grant G: The cytoarchitectonic organization of the spinal cord in the rat. I. The lower thoracic and lumbosacral cord. J Comp Neurol 1984, 230:133-141.

27. Zhuang Z-Y, Wen Y-R, Zhang D-R, Borsello T, Bonny C, Strichartz GR, Decosterd I, Ji R-R: A peptide c-Jun N-terminal kinase (JNK) inhibitor blocks mechanical allodynia after spinal nerve ligation: respective roles of JNK activation in primary sensory neurons and spinal astrocytes for neuropathic pain development and maintenance. Journal of Neuroscience 2006, 26:3551-3560.

28. Le Bars $D$, Chitour D, Clot AM: The encoding of thermal stimuli by diffuse noxious inhibitory controls (DNIC). Brain Res 1981, 230:394-399.

29. Fujii K, Motohashi K, Umino M: Heterotopic ischemic pain attenuates somatosensory evoked potentials induced by electrical tooth stimulation: diffuse noxious inhibitory controls in the trigeminal nerve territory. Eur J Pain 2006, 10:495-504.

30. Falinower S, Willer JC, Junien JL, Le Bars D: A C-fiber reflex modulated by heterotopic noxious somatic stimuli in the rat. J Neurophysiol 1994, 72:194-213.

31. Murase K, Kawakita K: Diffuse noxious inhibitory controls in antinociception produced by acupuncture and moxibustion on trigeminal caudalis neurons in rats. Jpn J Physiol 2000, 50:133-140.

32. Han JS, Terenius L: Neurochemical basis of acupuncture analgesia. Annu Rev Pharmacol Toxicol 1982, 22:193-220.

33. He LF: Involvement of endogenous opioid peptides in acupuncture analgesia. Pain 1987, 31:99-121.

34. Mayer DJ: Biological mechanisms of acupuncture. Prog Brain Res 2000, 122:457-477

35. Le Bars D, Chitour D, Kraus E, Dickenson AH, Besson JM: Effect of naloxone upon diffuse noxious inhibitory controls (DNIC) in the rat. Brain Res 1981, 204:387-402

36. Le Bars D, Willer JC, De Broucker T: Morphine blocks descending pain inhibitory controls in humans. Pain 1992, 48:13-20.

37. Jean A, Conductier G, Manrique C, Bouras C, Berta P, Hen R, Charnay Y, Bockaert J, Compan V: Anorexia induced by activation of serotonin 5-HT4 receptors is mediated by increases in CART in the nucleus accumbens. Proc Natl Acad Sci USA 2007, 104:16335-16340.

38. Bao H, Zhou Z, Yu Y, Han J: [C fiber is not necessary in electroacupuncture analgesia, but necessary in diffuse noxious inhibitory controls (DNIC)]. Zhen Ci Yan Jiu 1991, 16:120-124

39. Pomeranz B, Chiu D: Naloxone blockade of acupuncture analgesia: endorphin implicated. Life Sci 1976, 19:1757-1762.

40. Mayer DJ, Price DD, Rafii A: Antagonism of acupuncture analgesia in man by the narcotic antagonist naloxone. Brain Res 1977, 121:368-372.

41. Das S, Chatterjee TK, Ganguly A, Ghosh JJ: Role of adrenal steroids on electroacupuncture analgesia and on antagonising potency of naloxone. Pain 1984, 18:135-143.

42. Bossut DF, Huang ZS, Sun SL, Mayer DJ: Electroacupuncture in rats: evidence for naloxone and naltrexone potentiation of analgesia. Brain Res 1991, 549:36-46.

43. Kwon YB, Kang MS, Han HJ, Beitz AJ, Lee JH: Visceral antinociception produced by bee venom stimulation of the Zhongwan acupuncture point in mice: role of alpha(2) adrenoceptors. Neurosci Lett 2001, 308:133-137.

44. Koo ST, Park Yl, Lim KS, Chung K, Chung JM: Acupuncture analgesia in a new rat model of ankle sprain pain. Pain 2002, 99:423-431.

45. McLennan $H$, Gilfillan $K$, Heap Y: Some pharmacological observations on the analgesia induced by acupuncture in rabbits. Pain 1977, 3:229-238.

46. Chapman CR, Colpitts YM, Benedetti C, Kitaeff R, Gehrig JD: Evoked potential assessment of acupunctural analgesia: attempted reversal with naloxone. Pain 1980, 9:183-197.

47. Chapman CR, Benedetti C, Colpitts YH, Gerlach R: Naloxone fails to reverse pain thresholds elevated by acupuncture: acupuncture analgesia reconsidered. Pain 1983, 16:13-31.

48. Koo ST, Lim KS, Chung K, Ju H, Chung JM: Electroacupuncture-induced analgesia in a rat model of ankle sprain pain is mediated by spinal alpha-adrenoceptors. Pain 2008, 135:11-19.

49. Rittner HL, Labuz D, Schaefer M, Mousa SA, Schulz S, Schafer M, Stein C, Brack A: Pain control by CXCR2 ligands through $\mathrm{Ca}$ 2+-regulated release of opioid peptides from polymorphonuclear cells. Faseb J 2006, 20:2627-2629.

50. Stein C, Schafer M, Machelska H: Attacking pain at its source: new perspectives on opioids. Nat Med 2003, 9:1003-1008.

51. Machelska H, Schopohl JK, Mousa SA, Labuz D, Schafer M, Stein C: Different mechanisms of intrinsic pain inhibition in early and late inflammation. $J$ Neuroimmunol 2003, 141:30-39.

52. Tyce GM, Yaksh TL: Monoamine release from cat spinal cord by somatic stimuli: an intrinsic modulatory system. J Physiol 1981, 314:513-529.

53. Men DS, Matsui Y: Activation of descending noradrenergic system by peripheral nerve stimulation. Brain Res Bull 1994, 34:177-182.

54. Nuseir K, Proudfit HK: Bidirectional modulation of nociception by GABA neurons in the dorsolateral pontine tegmentum that tonically inhibit spinally projecting noradrenergic A7 neurons. Neuroscience 2000, 96:773-783.

55. Reddy SV, Maderdrut JL, Yaksh TL: Spinal cord pharmacology of adrenergic agonist-mediated antinociception. J Pharmacol Exp Ther 1980, 213:525-533.

56. Yaksh TL: Pharmacology of spinal adrenergic systems which modulate spinal nociceptive processing. Pharmacol Biochem Behav 1985, 22:845-858.

57. Eisenach JC, Detweiler DJ, Tong C, D'Angelo R, Hood DD: Cerebrospinal fluid norepinephrine and acetylcholine concentrations during acute pain. Anesth Analg 1996, 82:621-626.

58. Shinomura T, Nakao S, Adachi T, Shingu K: Clonidine inhibits and phorbol acetate activates glutamate release from rat spinal synaptoneurosomes. Anesth Analg 1999, 88:1401-1405.

59. Kawasaki $Y$, Kumamoto E, Furue $H$, Yoshimura M: Alpha 2 adrenoceptormediated presynaptic inhibition of primary afferent glutamatergic transmission in rat substantia gelatinosa neurons. Anesthesiology 2003 98:682-689.

60. Wei H, Pertovaara A: Spinal and pontine alpha2-adrenoceptors have opposite effects on pain-related behavior in the neuropathic rat. Eur J Pharmacol 2006, 551:41-49.

61. Fleetwood-Walker SM, Hope PJ, Mitchell R: Antinociceptive actions of descending dopaminergic tracts on cat and rat dorsal horn somatosensory neurones. J Physiol 1988, 399:335-348.

62. Millan MJ: Descending control of pain. Prog Neurobiol 2002, 66:355-474

63. Vanegas $H$, Schaible HG: Descending control of persistent pain: inhibitory or facilitatory? Brain Res Brain Res Rev 2004, 46:295-309.

64. Woolf CJ, Salter MW: Neuronal plasticity: increasing the gain in pain Science 2000, 288:1765-1769.

65. Appelbaum BD, Holtzman SG: Characterization of stress-induced potentiation of opioid effects in the rat. J Pharmacol Exp Ther 1984, 231:555-565

66. Amit Z, Galina ZH: Stress-induced analgesia: adaptive pain suppression. Physiol Rev 1986, 66:1091-1120.

67. Calcagnetti DJ, Holtzman SG: Factors affecting restraint stress-induced potentiation of morphine analgesia. Brain Res 1990, 537:157-162.

68. Terman GW, Liebeskind JC: Relation of stress-induced analgesia to stimulation-produced analgesia. Ann N Y Acad Sci 1986, 467:300-308,

69. Takeshige C, Kobori M, Hishida F, Luo CP, Usami S: Analgesia inhibitory system involvement in nonacupuncture point-stimulation-produced analgesia. Brain Res Bull 1992, 28:379-391.

70. Lin JG, Lo MW, Wen YR, Hsieh CL, Tsai SK, Sun WZ: The effect of high and low frequency electroacupuncture in pain after lower abdominal surgery. Pain 2002, 99:509-514.

doi:10.1186/1423-0127-17-19

Cite this article as: Wen et al: DNIC-mediated analgesia produced by a supramaximal electrical or a high-dose formalin conditioning stimulus: roles of opioid and $\alpha 2$-adrenergic receptors. Journal of Biomedical Science 2010 17:19. 